\title{
First detection of a G1-like H9N2 virus in Russia, 2018
}

\author{
Kirill Sharshov ${ }^{1, *}$, Olga Kurskaya ${ }^{1}$, Ivan Sobolev ${ }^{1}$, Sergey Leonov ${ }^{2}$, Marsel Kabilov ${ }^{3}$, Alikina Tatyana ${ }^{3}$, \\ Alexander Alekseev ${ }^{1}$, Anastasiya Derko ${ }^{1}$, Yuriy Yushkov ${ }^{2}$, Takehiko Saito ${ }^{4}$, Yuko Uchida ${ }^{4}$, Junki Mine ${ }^{4}$, \\ Victor Irza ${ }^{5}$, Alexander Shestopalov ${ }^{1}$ \\ ${ }^{1}$ Federal Research Center of Fundamental and Translational Medicine (CFTM), Novosibirsk 630117, Russia \\ ${ }^{2}$ Siberian Federal Scientific Centre of Agro-BioTechnologies, Krasnoobsk 630501, Russia \\ ${ }^{3}$ SB RAS Genomics Core Facility, Institute of Chemical Biology and Fundamental Medicine, Novosibirsk 630090, Russia \\ ${ }^{4}$ Division of Transboundary Animal Disease, National Institute of Animal Health, NARO, Tsukuba 305-0856, Japan \\ ${ }^{5}$ Federal Centre for Animal Health, Vladimir, Russia
}

\begin{abstract}
Worldwide, avian influenza H9N2 viruses of different lineages are the most widespread viruses in poultry. However, to date, cases in Russia have not been documented. In this study, we report the first detection of a G1-like H9N2 virus from poultry sampled at live-bird markets in Russia (Far East region) during the winter of 2018 (isolate A/chicken/Amur_Russia/17/2018). We assume there has been further circulation of the A/chicken/Amur_Russia/17/2018 H9N2 virus in the Russian Far East with possible distribution to other regions or countries in 2018-2019.
\end{abstract}

Keywords: H9N2 virus, genotype, poultry, Far East, Russia

*Corresponding author

Kirill Sharshov

Federal Research Center of Fundamental and Translational Medicine (CFTM),

Timakova St., Bldg. 2, Novosibirsk

630117, Russia

E-mail: sharshov@yandex.ru

ORCID:

Kirill Sharshov

https://orcid.org/0000-0002-3946-9872

Olga Kurskaya

https://orcid.org/0000-0002-1931-2026

Ivan Sobolev

https://orcid.org/0000-0002-4561-6517

Marsel Kabilov

https://orcid.org/0000-0003-2777-0833

Takehiko Saito

https://orcid.org/0000-0002-9633-5897

Yuko Uchida

https://orcid.org/0000-0002-5833-5917

Junki Mine

https://orcid.org/0000-0002-1603-8471

Alexander Shestopalov

https://orcid.org/0000-0002-9734-0620

Conflict of Interest

The authors declare no conflicts of interest.

Received: January 4, 2019

Revised: March 9, 2019

Accepted: March 11, 2019
Influenza H9N2 viruses have been detected in poultry over a wide area of East Asia, Middle East, Europe, and Southern Africa [1-4]. Multiple genotypes have been circulating in domestic birds in Mainland China since 1994, and H9N2 viruses are the most prevalent subtype of avian influenza viruses (AIVs) in chicken populations [5, 6].

The H9N2 viruses increase their phylogenetic diversity by undergoing extensive reassortments with other influenza viruses resulting in genetic reassortants with highly pathogenic avian influenza (HPAI) viruses including the H5N1 virus [7, 8]. Despite low pathogenicity in poultry, documented direct transmission of HXNX to humans raises concern about the pandemic potential of the H9N2 virus [9].

Based on hemagglutinin (HA) sequences, the H9N2 viruses from Asia, Europe, and Africa have been grouped into several basic genotypes represented by their prototype strains: A/quail/Hong Kong/G1/97 (G1-like), A/duck/ Hong Kong/Y280/9 (Y280-like), A/Chicken/Beijing/1/94 (BJ94-like), and A/ chicken/Korea/38349-P96323/96 (Korean-like) [7].

Although the H9N2 virus has been endemic in poultry populations for decades, its epidemiology and transmission pathways have not been fully described. Wild bird migration and poultry product movements are considered the main sources of transmission to novel regions $[2,4,10]$. The Far East region (FER) of Russia has had an important role in the persistence and distribution of AIV [11]. The FER is closely connected to the East Asian-Australasian bird flyway, and wild birds migrate to and congregate in FER wetlands for breeding and molting. The ecosystem features of the FER have allowed for the broadening of the distribution of HPAI viruses as evidenced by the spread of H5N1 clade 2.3.2 in 2008 [11]. Moreover, the FER of Russia has large numbers of poultry farms and backyard farms, which can increase the possibility of AIV transmission among different avian species. Until the winter of 2018, H9N2 viruses had not been documented in either in poultry or wild birds in Russia. Herein, we report the first detection of a G1like H9N2 virus in Russia in 2018. 
We collected oropharyngeal and cloacal swab specimens from chickens (Gallus gallus domesticus; $\mathrm{n}=97$ ) as well as environmental samples such as water and feces $(n=100)$. We tested all samples by using real-time reverse transcription polymerase chain reaction (RT-PCR; AmpliSens influenza virus À/Mgene-FRT PCR kit; InterLabService, Russia) after inoculation of viral samples into 10-day-old embryonated chicken eggs. The presence of influenza isolates in egg allantoic fluid was identified by using HA assays and real-time RT-PCR targeting the $M$ gene in accordance with the World Organization of Animal Health (OIE) protocols (available at http://www.oie.int/international-standard-setting/terrestrialcode/). Identification and isolation of viruses were performed according to the standard protocols at the biosafety level (BSL) 3 Laboratory of the Federal Research Center of Fundamental and Translational Medicine (CFTM; Russia). The isolated strain A/chicken/Amur_Russia/17/2018 (Amur2018) has been stored in the depositary of the CFTM.

Complete genome sequencing of the Amur-2018 virus was performed by next-generation sequencing at the Institute of Chemical Biology and Fundamental Medicine, Siberian Branch of Russian Academy of Sciences (Russia) using an Illumina MiSeq sequencer (Illumina, USA) and a Nextera XT DNA Library Preparation kit (Illumina) according to manufacturer's instructions.

The data were analyzed by using CLC Genomics Workbench 8.5 (Qiagen, Netherlands). Nucleotide sequences have been deposited with the Global Initiative on Sharing All Influenza Data (GISAID) under number EPI_ISL_309322. Maximum-likelihood phylogenies of each gene segment (HA and neuraminidase [NA]) were generated with MEGA5 software [12]. We reconstructed the phylogenetic trees by using selected representative sequences of H9N2 viruses that shared high nucleotide similarity $(>98 \%)$ and were available in GenBank and GISAID. Bootstrap analysis (500 replicates) was used to infer the confidence values for the phylogenetic tree.

All animal experiments were approved by the Ethics Committee of the CFTM (No. 2017-16). To perform the intravenous pathogenicity index (IVPI) test of the virus, $0.1 \mathrm{~mL}$ of 1:10 dilutions of infectious allantoic fluids were intravenously inoculated into ten 6-week-old specific-pathogen-free chickens (White Leghorn). The IVPI was calculated according to an OIE standard protocol (available at http://www. oie.int/international-standard-setting/terrestrial-code/) and isolates with an IVPI $>1.2$ were determined to be HPAI. All 10 chickens displayed no clinical signs and survived. Additionally, sera were harvested from the inoculated survived chickens at 21 days post inoculation (dpi) for seroconversion confirmation by performing hemagglutination inhibition (HI) assays as described in the OIE Manual of Diagnostic Tests and Vaccines for Terrestrial Animals. All experiments and challenge studies using live viruses were conducted in a BSL 3 facility.

We assessed the pathogenicity of the virus using 9-weekold a mouse strain $(B A L B / c)$ mice. Mice were lightly anes- thetized with ether, then inoculated intranasally with $50 \mu \mathrm{L}$ of successive tenfold dilutions of viruses suspended in phosphate-buffered saline (PBS; $10^{-1}$ to $10^{-5} \log$-dilution series). During the 20-day experiments, infected mice were observed for weight loss and clinical scores such as ruffled fur, hunched posture, and shivering were recorded. The control group of mice was inoculated with PBS. We tested lung homogenates on 5 dpi using RT-PCR and cell culture infection (Madin-Darby canine kidney [MDCK] cells) to assess replicative activity. We also harvested sera from the inoculated mice at $21 \mathrm{dpi}$ to determine antibody titer by using the HI test described in the OIE Manual of Diagnostic Tests and Vaccines for Terrestrial Animals.

During the period September 2017 to February 2018, we conducted an AIV surveillance program on wild, synanthropic, and domestic birds in the Amur area of the Russian FER. Several isolates of the H4N6 low pathogenicity AIV (LPAIV) were isolated from wild ducks (information available upon request), but no $\mathrm{H} 5, \mathrm{H} 7$, or $\mathrm{H} 9$ subtypes were detected. No outbreaks of HPAI have been reported in this region. Additionally, we investigated poultry to determine the presence of AIV infections in the Amur region. For this aim, we collected samples from chickens $(n=97)$, as well as environmental (water and feces) samples $(\mathrm{n}=100)$, in 2 live-bird markets and backyard poultry areas in countryside areas of the Amur region in February 2018.

Three samples obtained via oropharyngeal swabs and examined by using real-time RT-PCR were positive for AIV virus. Based on examining the results from AIV-inoculated chicken embryos, among the 3 positives, we only isolated 1 AIV isolate from a backyard chicken; subsequently, it was formally designated as A/chicken/Amur_Russia/17/2018 (herein, Amur-2018), and stored in our laboratory collection. Complete genome sequencing and phylogenetic analysis were performed as described above.

Based on the sequencing results, the Amur-2018 isolate was identified as an H9N2 subtype and was considered an LPAIV as it had an IVPI of 0.0 in chickens and an amino acid sequence representative of the HA proteolytic cleavage site (PARSSR/GLF). Homology basic local alignment search tool (BLAST) searches showed that the Amur-2018 isolate had all 8 RNA segments of the G1-like H9N2 genotype and had $>99 \%$ similarity to the H9N2 strains isolated in Israel in 2006-2007 (Table 1).

Phylogenetic analysis demonstrated that the HA (Fig. 1) and other genes of the Amur-2018 isolate belong to the G1like H9N2 phylogenetic group and Amur-2018 clusters with the H9N2 viruses identified earlier in the Middle East and Northern Africa [1, 3, 4]. Intriguingly, BLAST results showed a high similarity of Amur-2018 to A/chicken/Israel/215/2007, a strain that, since 2008, has been used as a vaccine strain [1, 13].

At the HA receptor binding site, the Amur-2018 isolate carried a Q226 substitution suggesting an affinity to avianlike receptors, whereas, it had glycine at position 228, indicating human virus-like cell tropism [5, 14]. No mutations 
Table 1. Nucleotide identity between A/chicken/Amur_Russia/17/2018 (H9N2) virus from Amur Region, Far East, Russia, and their near homologues in GenBank as of October $\overline{1}$, 2018

\begin{tabular}{llllc}
\hline \hline Gene & \multicolumn{1}{c}{ Virus } & Subtype & GenBank accession \# & Identity (\%) \\
\hline$P B 2$ & A/chicken/Israel/54/2008 & (H9N2) & FJ464699 & 99.77 \\
$P B 1$ & A/chicken/Israel/402/2007 & (H9N2) & FJ464682 & 99.78 \\
$P A$ & A/chicken/Israel/1638/2006 & (H9N2) & FJ464665 & 99.90 \\
$H A^{*}$ & A/chicken/Israel/386/2007 & (H9N2) & FJ464720 & 99.69 \\
& A/teal/Primorie/3628/02 & (H9) & DQ787797 & 83.64 \\
& A/teal/Primorie/3631/02 & (H9) & DQ787802 & 83.52 \\
$N P$ & A/chicken/Israel/386/2007 & FJ464632 & 99.78 \\
$N A$ & A/turkey/Israel/1608/2006 & (H9N2) & FJ464629 & 99.70 \\
$M P$ & A/chicken/Israel/215/2007 & FJ464598 & 99.90 \\
$N S$ & A/chicken/Israel/215/2007 & (H9N2) & FJ464649 & 99.63 \\
\hline
\end{tabular}

HA, hemagglutinin; MP, matrix; NA, neuraminidase; NP, nucleoprotein; NS, nonstructural; PA, polymerase acidic; PB1 and 2, polymerase basic 1 and 2 .

${ }^{*} H A$ of near homologue and the only 2 H9 strain previously isolated in Far East, Russia.

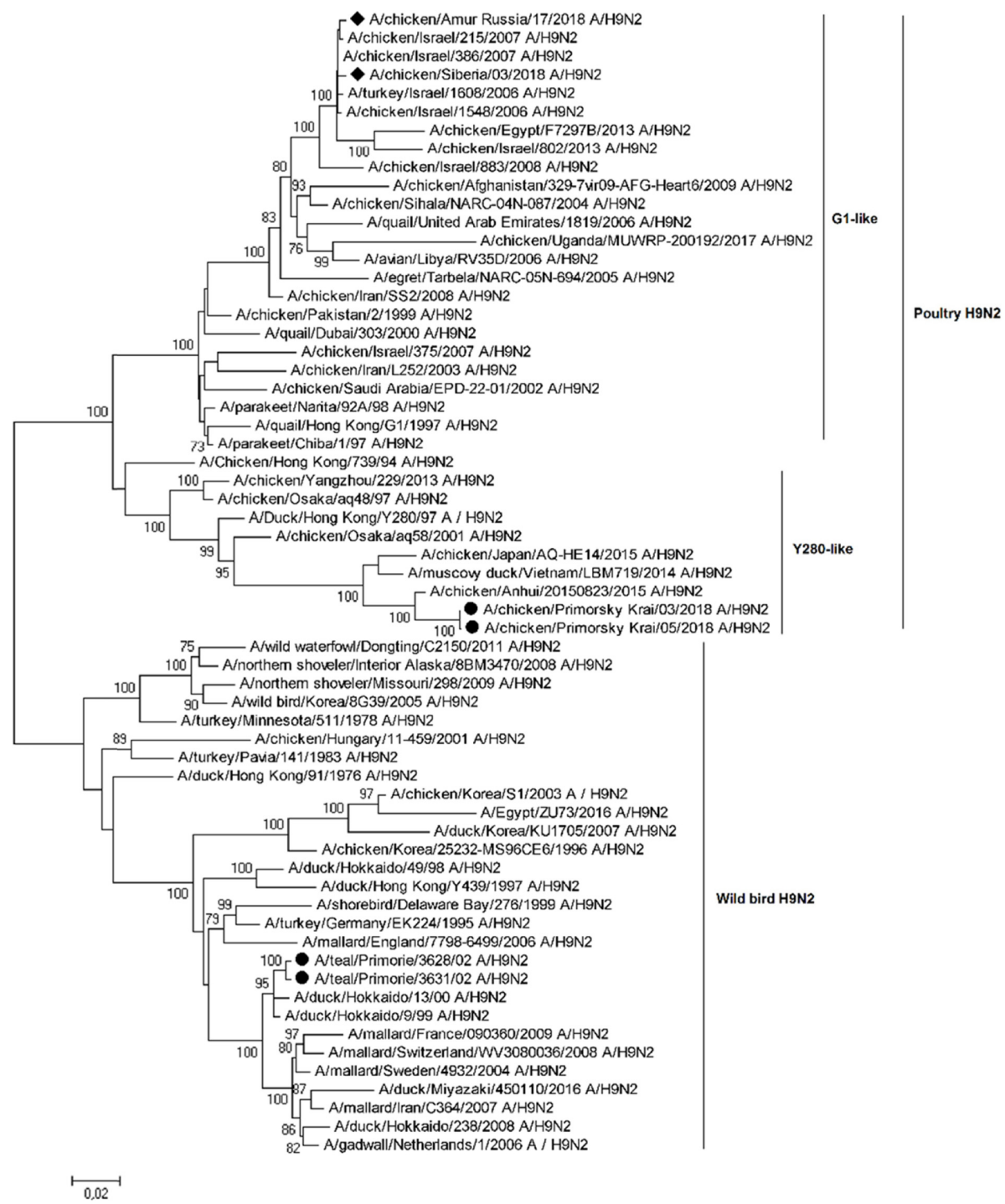

Fig. 1. Maximum-likelihood phylogenetic tree (GTR model) for the $H A$ gene. Bootstrap values were calculated based on 500 replicates. At each branch, the number indicates a bootstrap value ( $>70 \%)$. Black diamonds indicate the Russian H9N2 viruses isolated in 2002 and 2018. Scale bar indicates nucleotide substitutions per site. 
Table 2. Amino acid substitutions of A/chicken/Amur_Russia/17/2018 (H9N2) affecting the characteristics of the virus

\begin{tabular}{|c|c|c|c|c|}
\hline Protein & Position & Amino acid & Effect & Reference \\
\hline \multirow{2}{*}{ HA } & 226 & $\mathrm{Q}$ & Affinity to avian-like receptors & {$[5,14]$} \\
\hline & 228 & G & Human virus-like cell tropism & [5] \\
\hline NA & $62-64$ & Deletion* $^{*}$ & Virus adaptation from wild birds to chickens & [3] \\
\hline \multirow{2}{*}{ PB2 } & 627 & $\mathrm{~V}$ & Replication in mammalian cells and virulence in mice & [16] \\
\hline & 701 & $\mathrm{D}$ & Avirulent variant & {$[3,17]$} \\
\hline PB1 & 13 & $\mathrm{P}$ & Human-specific amino acid & {$[3,17]$} \\
\hline PA & 409 & $\mathrm{~S}$ & Avian-specific amino acid & {$[3,17]$} \\
\hline M2 & 31 & $\mathrm{~N}^{*}$ & Amantadine resistance & [9] \\
\hline NS1 & 149 & A & Associated with increased virulence of the H9N2 viruses in mammals & [3] \\
\hline
\end{tabular}

HA, hemagglutinin; NA, neuraminidase; PB1, polymerase basic 1; PB2, polymerase basic 2; PA, polymerase acidic; M2, Matrix-2; NS1, NS1 influenza protein.

*Means that mutation was not detected in Amur-2018.

were observed in the polymerase basic (PB) 2 protein at the D701 amino acid residue, thus retaining avian characteristics, whereas, at position 627 we detected valine (V) [3]. Substitutions of $627 \mathrm{~K}$ and $701 \mathrm{~N}$ have been associated with virulence and virus transmission in mammals [15]. The $627 \mathrm{~V}$ substitution indicates an increase in $\mathrm{H} 5 \mathrm{~N} 1$ virus replication in mammalian cells and virulence in mice when compared with the 627E strains [16]. Human-specific amino acid was observed at $13 \mathrm{P}$ in $\mathrm{PB} 1$ but not at $409 \mathrm{~N}$ in polymerase acidic (Amur-2018 possessed 409S) [3]. The NS1 gene contained $149 \mathrm{~A}$, which is associated with increased virulence of the H9N2 viruses in mammals [17]. The Amur-2018 virus did not possess $31 \mathrm{~N}$ in the $M 2$ gene, suggesting the presence of amantadine resistance [9]. No deletion suggesting potential virus adaptation from wild birds to chickens was observed at positions 62-64 of NA [17]. A summary of the main amino acid substitutions is presented in Table 2 .

We also assessed the pathogenicity of the Amur-2018 isolate in 9-week-old BALB/c mice. Treated mice did not lose weight and had no signs of respiratory disease; however, there were significant antibody titers in the HI test results after $21 \mathrm{dpi}$ (geometric mean titer was $367.09 \pm 6.96$ ). The Amur-2018 virus was detected in mouse lungs at 5 dpi by using RT-PCR (cycle threshold value, 22.6 \pm 2.9 ) as well as in inoculated MDCK cell cultures (mean titer, 3.1 \pm 0.2 $\log _{10} \mathrm{CID}_{50}$ ). In addition, the chicken IVPI results showed that the Amur-2018 isolate displayed low pathogenicity. In addition, HI test results showed there was significant seroconversion in chickens at $21 \mathrm{dpi}$ (geometric mean titer was 1,016.93 \pm 1.59 ). These observations suggest the Amur-2018 virus has high immunogenicity and has a potential for application in vaccine design [13].

The origin and source of the Amur-2018 virus remain unknown. However, we suggest 3 possible pathways for the spreading of a G1-like H9N2 virus to FER, Russia. The first is associated with wild bird migration, which is suspected to have had a key role in the emergence of LPAIV, particularly H9N2 [2, 4]. Ecologically, the Russian FER is connected to neighboring regions and countries via the migration of wild birds. However, until recently, no G1-like H9N2 virus was detected in wild birds in this region of Russia. Previously in this area, only 2 strains, A/teal/Primorie/3631/02 (H9N2; GenBank DQ787802) and A/teal/Primorie/3628/02 (H9N2; GenBank DQ787797) were isolated, both in 2002. The indicated strains are closely related to low pathogenicity classical avian-like viruses isolated from ducks in Japan, Korea, and Vietnam. However, they do not cluster with the main genotypes of H9N2 viruses that are endemic worldwide in domestic poultry. The Amur-2018 strain has some identity to previous Russian H9 viruses obtained from wild birds, but the identity similarity is only $83 \%$ (Table 1 ). In addition to the isolation of Amur-2018 in the winter of 2018, 2 Y280like viruses were isolated in the Primorsky region of Russia in May 2018 (Fig. 1); the second isolation of H9N2 from poultry in Russia (unpublished sequences have been released in GISAID by Marchenko et al.) It is known that the main genotypes of $\mathrm{G} 1$ and $\mathrm{Ck} / \mathrm{Bei}$-like $\mathrm{H} 9$ viruses are endemic for chickens and turkeys, but they have not been detected in wild birds. Furthermore, only classical H9N2 wild duck-like viruses are reported to actively circulate in wild birds in neighboring Japan and Korea $[18,19]$. Thus, we suggest a low likelihood for the H9N2 Amur-2018 virus to have invaded the FER via bird migration.

The second possible transmission pathway is related to poultry product transportation (e.g., live poultry, meat, or feed) from affected areas. Such cases have been previously documented for the H9N2 virus [10]. Meanwhile, the prevalence of different multiple genotypes of H9N2 in neighbor China has been reported [6, 7]. In addition, several cases of H9N2 were detected in Korea and Japan in 2016-2017 [18, 19]. However, in neighboring Korea, the 'Y439-like' or 'Korean like' H9N2 virus is currently more common. In this study, the isolated Arum-2018 was a G1-like H9N2 containing all 8 segments closely related to Middle East (Israel) strains, and it was not clustered with Asian viruses isolated in 2016-2017. Those observations lead to the suggestion that Amur-2018like viruses may have emerged from a western region of Russia. 
The third most possible pathway is related to the illegal usage of cheap unregistered vaccines by private farmers in the FER of Russia. Such vaccines may not be completely inactivated, which can lead to virus distribution as has been described for Newcastle disease vaccine [20]. Illegal imports of unregistered vaccines have occurred in some countries after antigenic drift of field viruses rendered the existing poorly matched vaccines less effective. There are no published reports on illegal influenza vaccination with illegally imported vaccines; however, illegal vaccination against avian influenza in areas where the use of avian influenza vaccines is prohibited has been described by OIE-FAO Network of Expertise on Animal Influenza (2013). Thus, there should be concern about the limited availability of information on the effects of illegal vaccination. The high similarity between Amur-2018 and the G1-like vaccine strain that has been in use since $2008[1,13]$ further indicates that the use of cheap unregistered vaccines may be a logical pathway for the spread of a G1-like H9N2 virus (Amur-2018) to FER, Russia.

Based on our observations, we assume there will be further circulation of H9N2 Amur-2018-like viruses in the Russian FER, and it may spread to other neighboring regions or countries. These findings emphasize the importance of AIV surveillance in the Asian region of Russia and indicate the importance of controlling the import of poultry products and illegal vaccines as potential pathways for the further emergence of H9N2 influenza viruses.

\section{Acknowledgments}

The study was supported by a grant of Russian Scientific Foundation (project \# 17-44-07001). We acknowledge the authors, originating and submitting laboratories of the sequences from GISAID's EpiFlu ${ }^{\mathrm{TM}}$ Database on which this research is based. Contact details of submitters can be found at: http://platform.gisaid.org/epi3/frontend\#39414f.

\section{References}

1. Davidson I, Fusaro A, Heidari A, Monne I, Cattoli G. Molecular evolution of H9N2 avian influenza viruses in Israel. Virus Genes 2014;48:457-463.

2. Hu M, Jin Y, Zhou J, Huang Z, Li B, Zhou W, Ren H, Yue J, Liang L. Genetic characteristic and global transmission of influenza A H9N2 virus. Front Microbiol 2017;8:2611.

3. Kandeil A, El-Shesheny R, Maatouq AM, Moatasim Y, Shehata MM, Bagato O, Rubrum A, Shanmuganatham K, Webby RJ, Ali MA, Kayali G. Genetic and antigenic evolution of H9N2 avian influenza viruses circulating in Egypt between 2011 and 2013. Arch Virol 2014;159:2861-2876.

4. Nagy A, Mettenleiter TC, Abdelwhab EM. A brief summary of the epidemiology and genetic relatedness of avian influenza H9N2 virus in birds and mammals in the Middle East and North Africa. Epidemiol Infect 2017;145:3320-3333.

5. Ge FF, Zhou JP, Liu J, Wang J, Zhang WY, Sheng LP, Xu F, Ju HB, Sun QY, Liu PH. Genetic evolution of H9 subtype influenza viruses from live poultry markets in Shanghai,
China. J Clin Microbiol 2009;47:3294-3300.

6. Wei Y, Xu G, Zhang G, Wen C, Anwar F, Wang S, Lemmon G, Wang J, Carter R, Wang M, Sun H, Sun Y, Zhao J, Wu $\mathrm{G}$, Webster RG, Liu J, Pu J. Antigenic evolution of H9N2 chicken influenza viruses isolated in China during 2009-2013 and selection of a candidate vaccine strain with broad crossreactivity. Vet Microbiol 2016;182:1-7.

7. Guan Y, Shortridge KF, Krauss S, Webster RG. Molecular characterization of $\mathrm{H} 9 \mathrm{~N} 2$ influenza viruses: were they the donors of the "internal genes of $\mathrm{H} 5 \mathrm{~N} 1$ viruses in Hong Kong? Proc Natl Acad Sci U S A 1999;96:9363-9367.

8. Li X, Liu B, Ma S, Cui P, Liu W, Li Y, Guo J, Chen H. High frequency of reassortment after co-infection of chickens with the H4N6 and H9N2 influenza A viruses and the biological characteristics of the reassortants. Vet Microbiol 2018;222:11-17.

9. Lin YP, Shaw M, Gregory V, Cameron K, Lim W, Klimov A, Subbarao K, Guan Y, Krauss S, Shortridge K, Webster R, Cox N, Hay A. Avian-to-human transmission of H9N2 subtype influenza A viruses: relationship between H9N2 and H5N1 human isolates. Proc Natl Acad Sci U S A 2000;97: 9654-9658.

10. Mase $M$, Eto $M$, Imai $K$, Tsukamoto $K$, Yamaguchi $S$. Characterization of H9N2 influenza A viruses isolated from chicken products imported into Japan from China. Epidemiol Infect 2007;135:386-391.

11. Lvov DK, Shchelkanov MY, Prilipov AG, Vlasov NA, Fedyakina IT, Deryabin PG, Alkhovsky SV, Grebennikova TV, Zaberezhny AD, Suarez DL. Evolution of highly pathogenic avian influenza $\mathrm{H} 5 \mathrm{~N} 1$ virus in natural ecosystems of northern Eurasia (2005-08). Avian Dis 2010;54:483-495.

12. Tamura K, Peterson D, Peterson N, Stecher G, Nei M, Kumar S. MEGA5: molecular evolutionary genetics analysis using maximum likelihood, evolutionary distance, and maximum parsimony methods. Mol Biol Evol 2011;28:27312739

13. Wang Y, Davidson I, Fouchier R, Spackman E. Antigenic cartography of $\mathrm{H} 9$ avian influenza virus and its application to vaccine selection. Avian Dis 2016;60:218-225.

14. Matrosovich MN, Krauss S, Webster RG. H9N2 influenza A viruses from poultry in Asia have human virus-like receptor specificity. Virology 2001;281:156-162.

15. Wang J, Sun Y, Xu Q, Tan Y, Pu J, Yang H, Brown EG, Liu J. Mouse-adapted H9N2 influenza A virus PB2 protein M147L and E627K mutations are critical for high virulence. PLoS One 2012;7:e40752.

16. Taft AS, Ozawa M, Fitch A, Depasse JV, Halfmann PJ, HillBatorski L, Hatta M, Friedrich TC, Lopes TJ, Maher EA, Ghedin E, Macken CA, Neumann G, Kawaoka Y. Identification of mammalian-adapting mutations in the polymerase complex of an avian H5N1 influenza virus. Nat Commun 2015;6:7491.

17. Lin $\mathrm{TN}$, Nonthabenjawan $\mathrm{N}$, Chaiyawong S, Bunpapong $\mathrm{N}$, Boonyapisitsopa S, Janetanakit T, Mon PP, Mon HH, Oo $\mathrm{KN}$, Oo SM, Mar Win M, Amonsin A. Influenza A(H9N2) virus, Myanmar, 2014-2015. Emerg Infect Dis 2017;23:10411043.

18. Kang M, Jang HK. Genetics and biological property analysis of Korea lineage of influenza A H9N2 viruses. Vet Microbiol 2017;204:96-103. 
42 Kirill Sharshov, Olga Kurskaya, Ivan Sobolev, Sergey Leonov, Marsel Kabilov, Alikina Tatyana, Alexander Alekseev, Anastasiya Derko, Yuriy Yushkov, Takehiko Saito, Yuko Uchida, Junki Mine, Victor Irza, Alexander

19. Okamatsu M, Sakoda Y, Kishida N, Isoda N, Kida H. Antigenic structure of the hemagglutinin of H9N2 influenza viruses. Arch Virol 2008;153:2189-2195.
20. Pedersen JC, Hines NL, Killian ML, Predgen AS, Schmitt BJ. Isolation of a virulent Newcastle disease virus from confiscated LaSota vaccine. Avian Dis 2013;57:316-320. 